\title{
HACIA LA ELIMINACIÓN DE LAS BRECHAS RACIALES: JUVENTUD Y PROGRAMAS DE LA REVOLUCIÓN
}

\author{
RODRIGO ESPINA PRIETO*
}

\begin{abstract}
RESUMEN
La política de la Revolución, desde el propio triunfo de 1959, ha ido probando la alta efectividad de las políticas universales de equidad para dinamizar procesos acelerados de movilidad social ascendente para los sectores populares y en particular, para el mejoramiento de los grupos raciales preteridos, mayoritarios, generalmente, dentro de estos sectores. A pesar de esto se mantienen brechas de equidad racializadas, asociadas a condiciones de partida desventajosas que suponen barreras de movilidad social. Esto ha implicado la necesidad de combinar instrumentos universales con acciones afirmativas integrales a las que responden, en gran medida, los programas quedan expuestos en el artículo.
\end{abstract}

\section{PALABRAS CLAVE: RELACIONES RACIALES, EQUIDAD RACIAL, POLÍTICAS DE JUVENTUD}

* Máster en Antropología. Investigador del Centro de Estudios Sobre la Juventud. Profesor de la Universidad de La Habana.

E-Mail: cestedit@jovenclub.cu. 


\title{
RUMO À ELIMINAÇÃO DAS LACUNAS RACIAIS: JUVENTUDE E PROGRAMAS DA REVOLUÇÃO
}

\begin{abstract}
RESUMO
A política da Revolução, desde o próprio triunfo em 1959, vem demonstrando a alta efetividade das políticas universais de eqüidade para dinamizar processos acelerados de mobilidade social ascendente para os setores populares, e em particular para a melhoria dos grupos raciais preteridos, geralmente majoritários dentro destes setores. Apesar disso, mantêm-se lacunas racializadas de eqüidade, associadas a condições iniciais desvantajosas que constituem barreiras de mobilidade social. Isto implica a necessidade de se combinar instrumentos universais com ações afirmativas integrais, conforme demonstram em grande parte os programas apresentados neste artigo.
\end{abstract}

PALAVRAS-CHAVE: RELAÇÕES RACIAIS, EQÜIDADE RACIAL,
POLÍTICAS DE JUVENTUDE

\section{TOWARDS THE ELIMINATION OF RACIAL GAPS: YOUTH AND PROGRAMS OF THE REVOLUTION}

\begin{abstract}
The policy of the Revolution, from the very triumph of 1959 has been testing the high effectiveness of policies to boost the universal equity accelerating processes of upward social mobility for the popular sectors and in particular for the improvement of disenfranchised racial groups, majority, usually within these sectors. Despite this equity gaps remain racialized, associated with unfavorable starting conditions involving social mobility barriers. This implied the need to combine universal instruments comprehensive affirmative action in response to a large extent, the programs are explained in the article.
\end{abstract}

KEY WORDS: RACE RELATIONSHIPS, RACIAL EQUITY, YOUTH POLICIES 
Para nosotros, los revolucionarios, luchar contra la discriminación racial es un privilegio sagrado. FIDEL CASTRO

\section{INTRODUCCIÓN}

DURANTE LOS ÚLTIMOS VEINTE años, el tema de las relaciones raciales -entendidas éstas como las formas específicas en que interactúan socialmente los individuos de diferente filiación racial, mediadas estas formas de integración por factores históricos, económicos y socioculturales, que determinan las particularidades que en cada sociedad y momento histórico se manifiesta el racismo en sus formas de discriminación racial y prejuicio racial (Pérez Álvarez,1996) - ha ido ocupando con fuerza los escenarios de discusión académica, los artísticoculturales (teatro, plástica, música, en particular el hip-hop), los escenarios políticos, con el tratamiento del tema por parte de Fidel Castro, máximo líder del proceso revolucionario que lleva a cabo Cuba desde 1959 (Castro, 2003 y 2006), y, también los escenarios barriales.

Un hecho es cierto, la identificación temprana del problema racial como uno de los primeros a los que debería dar solución la Revolución. El punto de partida del movimiento revolucionario para esta conclusión respondía a la situación desventajosa en que se encontraba la población no blanca - negra y mestiza - a partir del racismo implantado durante la colonia y mantenido durante medio siglo de república mediatizada en la isla.

Por lo tanto, la Revolución, desde 1959, emprendió el camino de profundas transformaciones, que indiscutiblemente beneficiaban a los sectores más populares de la población, en las que el racismo y sus bases socioeconómicas y culturales quedaron muy maltrechos. Entre ellas se pueden mencionar como las más importantes: la reforma urbana, la reforma agraria, la campaña de alfabetización y la universalización de la enseñanza gratuita a todos los menores de edad; el cubrimiento total de la población con los servicios gratuitos de salud; el pleno empleo, así como un grupo de acciones en este sentido para el beneficio de sectores particulares, como podían ser las domésticas, que pudieron abandonar dicha labor e incorporarse al trabajo en instituciones estatales; la eliminación, a través de la nacionalización (1961), de todos los exclusivismos raciales - y de otro tipo- que existían en clubes y asociaciones, transformaciones diri- 
gidas todas a la eliminación de cuanta diferencia existiera en la sociedad cubana.

La eliminación de la propiedad privada sobre los medios fundamentales de producción, provocó importantes cambios en la estructura socio clasista, con profundas implicaciones en el logro de la equidad racial limitando el ejercicio de la discriminación racial en centros laborales y propiciando la interrelación racial; no sólo en la esfera laboral, sino también en la social más general, con la creación de un conjunto de instituciones de carácter político-social: Comités de Defensa de la Revolución (CDR), Federación de Mujeres Cubanas (FMC), milicias, organizaciones estudiantiles, entre otras.

Aunque la Revolución no elaboró una ley específica contra la discriminación racial y el conjunto de leyes y medidas aplicadas de corte universalistas, presentan limitaciones de diferente orden para la solución de un problema de tal magnitud como es el de las relaciones raciales; puede afirmarse que la transformación profunda de la estructura socioclasista heredada a favor de las clases más desposeídas en general, lo que provocó que las desigualdades existentes entre los diferentes estratos de la población resultaran mínimas, la aplicación de políticas universales de equidad, o sea que no estaban dirigidas a un sector poblacional en particular, sino a toda la población en general; junto a un discurso político francamente desestructurador del racismo, que provenía de las principales figuras dirigentes del proceso revolucionario, socavaron las bases sociales e ideológicas del racismo y fueron limitando el ejercicio de la discriminación al plano de las relaciones interpersonales en su sentido más íntimo. A la par, habría que añadir la actuación de Cuba en el plano internacional, en particular en África, y su contribución a la lucha de los pueblos contra el racismo, entre otras formas de dominación y explotación.

A pesar de este proceso, en el entendido de la necesidad de unión de todo el pueblo en torno a la Revolución y de que el racismo existente era un racismo residual, ${ }^{1}$ que con el paso del tiempo y la implantación de las medidas socialistas, cada vez más profundamente revolucionarias, y la educación de las nuevas generaciones, iría desapareciendo; se fue conformando una zona de silencio, de invisibilidad, alrededor del tema, que lo fue convirtiendo en tabú. El tema se convirtió en inconveniente.

1 En general hubo una tendencia a no utilizar el término racismo, sino el de prejuicio con una intención clara de «rebajarle el perfil» al fenómeno. 
Es más, para muchos, el problema del racismo en Cuba era un problema ya solucionado en gran medida. Así en 1986 se publica El problema negro en Cuba y su solución definitiva, de Pedro Serviat (1986), en el que se argumenta la inexistencia del racismo en Cuba y su imposibilidad de resurgimiento, bajo las condiciones del socialismo.

Se trata de que en la lógica de la política social típica de la transición socialista cubana, el tema de la equidad entre los grupos de color de piel diferente, se manejó dentro de una variante de integración social general, con muy pocos instrumentos de políticas afirmativas, en el entendido de que si negros y mestizos formaban parte mayoritariamente de los sectores populares, las acciones de promoción de éstos tendrían un efecto directo y equiparable al esperado sobre el resto de los grupos. Con ello se trataba también de no extender y reforzar, con instrumentos focalizadores particulares, la estigmatización vinculada al color de la piel y de mantener como valor político supremo la unidad por sobre las diferencias.

Sin embargo, esa política racialmente indiferenciada, junto a condiciones de partida asimétricas de los grupos raciales, desventajosos para los sectores más humildes en los que estaban sobre representados los negros y mestizos, a pesar de los beneficios que reportaban para la población en general, fue estableciendo brechas en perjuicio precisamente de estos grupos más coloreados de la población.

En este sentido, en 1985 el Tercer Congreso del Partido Comunista de Cuba, a partir del desbalance existente en la proporcionalidad de los diferentes grupos raciales — así como de jóvenes y mujeresen la estructura de cuadros de dirección de diversos sectores económicos y políticos - fenómeno develado por el Censo Nacional de Población y Vivienda de 1981-, propone:

Una adecuada representación femenina acorde con la participación y el aporte importante de las mujeres a la construcción del socialismo en nuestro país, y la presencia de la creciente cantera de jóvenes y prometedores valores nacidos y forjados en la Revolución. La composición étnica ${ }^{2}$ de nuestro pueblo, unida al mérito revolucionario y al talento probado de muchos compatriotas, que en el pasado eran discriminados por el color de la piel, debe estar justamente representada en los cuadros dirigentes del PCC. [...] Esta promoción de todos los componentes y valores de nuestra sociedad, y su integración en el Partido y en su dirección, no pueden ser dejadas a la espontaneidad (Partido Comunista de Cuba, 1985).

2 El documento confunde lo étnico con lo racial. 
A partir de este análisis se estableció un sistema de cuotas para la promoción a cargos de dirección, que atendía a una tríada diferenciadora - joven, negro, mujer - , pero que en la práctica resultó formalista y limitado y provocó, en el aspecto racial, que muchos negros y mestizos, promovidos o no, se sintieran discriminados, pues en ocasiones esa sola condición sirvió para la promoción laboral o política, a contrapelo de lo planteado en el análisis. Además, en el propio análisis e implementación de esta medida, sólo se tuvieron en cuanta los problemas de representación estructural de forma limitada y no en toda su amplitud, así como tampoco los de carácter subjetivo, lo que implicó, en gran medida, su falta de efectividad.

No existió en su momento una discusión social de estas medidas del sistema de cuotas. Sus consecuencias en el sentido negativo, tuvieron una repercusión tardía y centrada únicamente en el medio académico e intelectual. Hay que reconocer que también hubo muchos jóvenes, mujeres y negros promovidos a cuadros dirigentes que pasaron «la prueba del tiempo», así como que la intencionalidad de esta medida se mantuvo y hoy día son miles los cuadros con esas condiciones que están en un proceso ascendente en la línea de dirección partidista y de los organismos de la administración central del Estado.

Este complejo proceso, someramente reseñado hasta aquí, se ve reflejado en la bibliografía sobre el tema que se publica en el período posterior a 1959 (Cuba, 1986). Si se realiza un ligero estudio infométrico, puede observarse cómo, si en los momentos iniciales del proceso el tema del racismo y sus correlatos psicológico y conductual -el prejuicio y la discriminación raciales - son las temáticas en que se enfatiza en las publicaciones cubanas, en el sentido de su existencia y del combate contra este fenómeno en las nuevas condiciones socioeconómicas que había creado la Revolución. En la medida en que avanza el proceso revolucionario, esta temática específica —en sus expresiones contemporáneas en Cuba - va desapareciendo y sólo se hace referencias a aspectos de carácter históricos, folklóricos y a los aportes del negro a la cultura, tanto nacional como latinoamericana. Esto sucede tanto en libros como en artículos, como el ya mencionado de Serviat, referido a la extinción del racismo en Cuba; situación que se extiende hasta finales de los 80 .

Ya a finales de esta década y fundamentalmente a principios de los 90, comienzan a desarrollarse un grupo importante de investigaciones, en particular las iniciadas por el Instituto Cubano de Antropología sobre la problemática de las relaciones raciales en Cuba. 
Los resultados finales de estas investigaciones fueron presentados a la comunidad científica. En ellas se constató la persistencia del racismo en Cuba - a pesar de las condiciones creadas para limitar su ejercicio-, no sólo como herencia, sino también como fenómeno asociado a las brechas que fue dejando la Revolución en este sentido y que en las condiciones de crisis en que se vio envuelto el país en los '90, se reproducen y se generan nuevos focos de irradiación del prejuicio racial y de la discriminación, en diferentes escenarios de la sociedad cubana.

A pesar de esta constatación y de la importancia que tiene el fenómeno racial en nuestra sociedad, no todas las investigaciones incorporaron el tema racial entre sus perspectivas. Hubo de pasar cierto tiempo, así como el reconocimiento por parte del discurso político del fenómeno del racismo, para que la variable racial comenzara a aparecer de forma integrada en el escenario de las investigaciones sociales cubanas.

\section{LA CRISIS DE LOS 90 Y EL TEMA DE LAS RELACIONES INTERRACIALES}

A partir de la crisis política sufrida por el campo socialista que implicó su desaparición, incluyendo a la Unión Soviética, se genera en el país una crisis con profundas implicaciones no sólo en el orden económico, sino también en la estructura socioclasista que desde 1959 se había ido gestando; este evento fue ampliando las diferentes brechas — en particular las raciales - no cerradas durante los períodos anteriores. Además, la reforma emprendida para enfrentar la crisis, produjo profundos cambios y fue creando nuevos ámbitos y escenarios - apertura al capital extranjero y ampliación del sector mixto de la economía, ampliación de las posibilidades del trabajo por cuenta propia, por citar sólo dos- en los que la brecha racial también fue adoptando nuevas formas de expresión.

En este sentido, como una síntesis de los resultados de diversas investigaciones y datos tomados de otras fuentes, pudieron apuntarse, entre otros, los siguientes aspectos.

Se manifiestan desproporciones en la representación de los grupos raciales entre los sectores emergente y tradicional de la economía. (Colectivo de Autores, 2003).

En el primero, el emergente, el más ventajoso por su asociación con la generación de divisas, entre otras posibilidades, existe una mayor representación de blancos en las categorías de dirigentes y profesionales 
y muy escasa la de los negros y mestizos, quienes sólo hallan una representación mayoritaria en su conjunto entre los trabajadores que prestan servicio indirecto al turismo (Colectivo de Autores, 2003).

Sin embargo, en el sector tradicional los negros y mestizos tienen una presencia significativa entre los profesionales y técnicos y es mayoritaria entre los obreros (Colectivo de Autores, 2003).

En relación con las remesas enviadas desde el exterior a familiares y amigos, el estudio constató que existen diferencias tanto entre los grupos raciales como los sociolaborales, pues las reciben más los trabajadores del sector emergente que los del tradicional; los grupos que se encuentran en los dos polos de la recepción son precisamente el de los profesionales del sector emergente y el de los obreros del sector tradicional con mayoría de negros y mestizos. Otro elemento importante, que tiene que ver con brechas regionales y territoriales, es que las remesas son recibidas en mayor cantidad por los sujetos de la muestra residentes en Ciudad de La Habana que por los de Santiago de Cuba. Todos estos aspectos están relacionados con la estructura racial histórica de la migración cubana (Colectivo de Autores, 2003).

En la ocupación del espacio urbano y la vivienda - como elementos representativos de las brechas raciales - se constata la mayor presencia de blancos en barrios residenciales y en viviendas con mejores condiciones habitacionales, y la presencia de negros y mestizos, (desde el punto de vista racial) y de obreros (en el ocupacional) en mayor proporción en los barrios populares y, además, en las viviendas de peores condiciones, fundamentalmente solares y ciudadelas. Este es un aspecto no siempre debidamente valorado más allá de las condiciones intrínsecas de la vivienda y de su situación territorial, sino que también brinda accesos a determinadas posibilidades que en la reforma económica se han abierto como son el «trabajo por cuenta propia, como autoempleo individual y como microempresas, fundamentalmente familiares, en actividades seleccionadas limitadas (servicios gastronómicos, alquiler de habitaciones, por ejemplo)» para los que una vivienda en mejores condiciones brinda mayores posibilidades (Colectivo de Autores, 2003).

En el plano de las relaciones interpersonales, ${ }^{3}$ en todas sus expresiones, aparecen signadas por el fenómeno de las relaciones raciales en

3 Se estudiaron las relaciones de pareja, las intrafamiliares, las de amistad y de vecindad, así como las que se establece entre condiscípulos y compañeros de trabajo. 
las que se dan dos tendencias básicas; en lo general, una a la intrarracialidad en todos los grupos raciales y otra a la preferencia de blancos para establecer dichas relaciones (Colectivo de Autores, 2003).

Diversos estudios parciales, aunque escasos aún, revelan la aguda sensibilidad que han desarrollado los jóvenes ante las desigualdades raciales, y su mayor desenfado ante las propuestas de una nueva socialización que le brindan las nuevas condiciones que ha ido gestando la sociedad cubana; por lo que - según la percepción de informantes de diferentes generaciones y color de piel- es más frecuente entre ellos establecer relaciones interpersonales más libres, espontáneas y desprejuiciadas, lo que implica que el problema va perdiendo significación al interior de las más nuevas generaciones, a la vez que están más advertidos de las diferentes formas que acusa este fenómeno (Izquierdo y Rodríguez, 2006; Colectivo de Autores, 2003).

Para finales de los años ochenta una mayor proporción de estudiantes negros terminaban sus estudios al finalizar noveno grado y los mestizos tenían una fuerte presencia en la enseñanza politécnica, en tanto los blancos eran mayoría entre los estudiantes universitarios. En los Institutos Preuniversitarios en el Campo (modalidad de estudios en el nivel medio superior que no exige altos desempeños meritocráticos para acceder a ella y de calidad comparativamente inferior en la preparación vocacional) la proporción de negros y mestizos ascendía a 51\%, mientras que en los Institutos Preuniversitarios Vocacionales de Ciencias Exactas (por el contrario a la modalidad anterior, de altas exigencias para el acceso y el rendimiento) eran el 40\% (Domínguez y Díaz, 1997).

No existen grandes diferenciales según color de la piel en cuanto a los niveles de instrucción alcanzados por la población en los últimos años. No obstante, se constatan diferencias significativas en el nivel educacional superior, donde los blancos culminan más estos estudios que los no blancos. 
Hacia la eliminación de las brechas raciales

TABLA 1: INDICADORES RELACIONADOS CON LA EDUCACIÓN, SEGÚN DATOS DEL CENSO DE POBLACIÓN Y VIVIENDAS, EN PORCENTAJES

\begin{tabular}{|l|c|c|c|c|}
\hline \multirow{2}{*}{ Nivel educacional } & \multirow{2}{*}{ Blancos } & \multicolumn{3}{|c|}{ No blancos } \\
\cline { 3 - 5 } & & Total & Negros & Mestizos \\
\hline Media poblacional & $\mathbf{6 5 , 1}$ & $\mathbf{3 4 , 9}$ & $\mathbf{1 0 , 1}$ & $\mathbf{2 4 , 8}$ \\
\hline Ninguno & 65,3 & 34,7 & 9,2 & 25,5 \\
\hline Primaria & 66,2 & 33,8 & 9,4 & 24,4 \\
\hline Secundaria básica & 63,8 & 36,2 & 10,6 & 25,6 \\
\hline Obrero calificado & 55,0 & 45,0 & 13,8 & 31,2 \\
\hline PreUniversitario & 63,9 & 36,1 & 11,3 & 24,8 \\
\hline Técnico medio & 64,5 & 35,5 & 11,6 & 23,9 \\
\hline Pedagogía nivel medio & 64,1 & 35,9 & 12,2 & 23,7 \\
\hline Superior o universitario & 69,5 & 30,5 & 10,7 & 19,8 \\
\hline
\end{tabular}

Fuente: Censo Nacional de Población y Viviendas, 2002.

A partir del procesamiento de los datos de las Encuestas de Hogares para Ciudad de La Habana, arrojó que los grupos que perciben los menores ingresos, están compuestos en proporción elevada por familias negras y mestizas; grupos en los que además, más del $25 \%$ de los integrantes de las familias tiene como promedio una escolaridad inferior a los 9 grados. En contraste, aproximadamente el 68\% de las familias de mayores ingresos son blancas, con una escolaridad alta (Añé, Ferriol y Ramos, 2004).

La ocupación por color de la piel de la población mayor de 15 años, no acusa diferencias acentuadas entre los diferentes grupos raciales, aunque los porcentajes de ocupación al interior de cada grupo $(97,11 \%, 97,01 \%, 96,68 \%$, para los blancos, los negros y los mestizos, respectivamente), presentan un mejor indicador para los blancos, en relación con cada uno de los otros grupos o con los dos en su conjunto (Colectivo de Autores, 2009).

TABLA 2: POBLACIÓN DE 15 AÑOS Y MÁS ECONÓMICAMENTE ACTIVA, POR SITUACIÓN DE ACTIVIDAD ECONÓMICA SEGÚN COLOR DE LA PIEL

\begin{tabular}{|l|c|c|c|c|c|cc|}
\hline Población & Cuba & Blancos & $\%$ & Negros & $\%$ & Mulatos o & $\%$ \\
\hline Total & 4.424 .650 & 2.829 .741 & 63,95 & 528.571 & 11,94 & 1.066 .338 & 24,09 \\
\hline Ocupados. & 4.291 .766 & 2.747 .970 & 64,02 & 512.809 & 11,94 & 1.030 .987 & 24,02 \\
\hline Desocupados & 132.884 & 81.771 & 61,53 & 15.762 & 11,86 & 35.351 & 26,60 \\
\hline
\end{tabular}

Fuentes: Censo Nacional de Población y Viviendas, 2002. 
Sin embargo, la composición racial de los ocupados por rama de actividad - de la cual sólo se obtuvo información para 1995- expresa una amplia participación de los blancos en todas las ramas, lo que es normal por ser la población de Cuba y de este grupo (PEA) mayoritariamente de color de piel blanca. En cambio, las estadísticas prueban que la generalidad de los hombres de color de piel negra se ubica fundamentalmente en la esfera de la construcción, transporte o comunicaciones. En el caso de las mujeres, la mayoría de las de color de piel negra trabajan en el sector agropecuario, transporte y comunicaciones, estando estas ramas entre las de peores remuneraciones. Y de donde se puede inferir mayor presencia de personas de color de piel negra en posiciones económicas desventajosas por la existencia de desigualdades en la tenencia de capacidades para el acceso a otras posiciones (Colectivo de Autores, 2009).

Estas informaciones indican que, no obstante los empíricamente apreciables impactos positivos de la concepción integradora y universalista de la política social sobre las desigualdades raciales; en una causalidad recursiva se han reproducido desventajas socioeconómicas entre grupos por color de la piel. Brechas heredadas, no atendidas anteriormente de forma diferenciada, manifiestan una reproducción que tiene en su base las dificultades y obstáculos que generan condiciones de partida asimétricas para aprovechar la equidad de oportunidades y revertirla en equidad de resultados, lo que se traduce - a contrapelo de las intenciones de la política social- en la persistencia y ampliación de desigualdades raciales de soporte estructural (desigualdades económicas racializadas) y simbólico (pervivencia de estereotipos, prejuicios y actitudes discriminatorias) que afectan a los grupos no blancos y en una acentuación de la articulación clase-raza. ${ }^{4}$

En términos de explicación de la movilidad y de barreras para acceder a sus corrientes ascendentes, y muy especialmente a las oportunidades de movilidad selectivas abiertas por la reforma, puede inferirse la presencia de capital humano y social y de activos tangibles e intangibles de menor competitividad en los grupos de negros y mestizos. La conservación - en proporción aún no determinada- de mecanismos de reproducción intergeneracional de estas carencias, pone en situación de desventaja a estos grupos ante instrumentos de distri-

4 Este es el término clásico en la sociología y la antropología, pero preferimos color de la piel, que hemos empleado en otros momentos, por la inoperancia científica del término raza para «clasificar» a las personas. 
bución mercantiles; lo que fortalece el peso de las condiciones iniciales de cada grupo social en el acceso desigual al bienestar, todo lo cual se entrelaza y refuerza con la persistencia de prejuicios raciales.

A pesar de que la crisis de los noventa afectó la calidad y posibilidades como satisfactores de necesidades de los servicios públicos, y de que varias de las medidas del reajuste económico implicaron el traspaso hacia el mercado de buena parte del consumo familiar, no se ha producido una disminución de la cobertura creada y los gastos sociales han tendido a recuperarse. De manera que es posible hablar de una reforma económica con apertura de mercado y ciertos grados de descentralización, pero que conserva y amplía una política social de equidad, de corte universal y unitaria, donde el Estado es el máximo responsable y actor.

No se cuenta aún con una base de datos sólida que permita evaluar los efectos del nuevo momento de la política social sobre la movilidad y la recuperación de la equidad, pero puede adelantarse que, - considerando el lugar privilegiado que se otorga en esta fase al acceso masivo a la enseñanza superior para los jóvenes y la total dislocación territorial de estas nuevas oportunidades de estudios- éste será el factor más dinámico de la movilidad, generando corrientes ascendentes en este particular; las que se refuerzan con el proceso de terciarización de la economía, donde los servicios especializados de alta calificación tienden a incrementarse (fundamentalmente los relacionados con la salud, el turismo y la informática).

\section{HACIA LA ELIMINACIÓN DE LAS BRECHAS RACIALES: JUVENTUD Y PROGRAMAS DE LA REVOLUCIÓN}

El enfrentamiento a la crisis implicó adoptar una nueva estrategia, gracias a la cual, dicha crisis se fue revertiendo paulatinamente desde los últimos años de la década de los 90 , y fue permitiendo al país recobrar su capacidad de enfrentar las políticas sociales, ahora con una nueva óptica, condicionada por la experiencia del período especial.

Los retos y dificultades que implica y deben enfrentar las políticas sociales, son muchos. En alguna medida inéditos para la sociedad cubana. Como efecto de su lógica interna de desarrollo, los cambios ocurridos a nivel mundial, así como por efecto del período de crisis, la sociedad cubana se ha diversificado, ha ampliado y profundizado sus intereses, anhelos y aspiraciones, ha abierto nuevas perspectivas al humanismo, a la solidaridad, al interior y hacia el exterior. Es más 
exigente consigo misma en materia de política social y en particular de las políticas dirigidas a las más nuevas generaciones de juventud.

Esta política social, que pudiera llamarse de nuevo tipo, iniciada a partir del año 2000, ha implicado la ejecución de un conjunto de programas sociales de beneficio fundamentalmente para los jóvenes, que superan en la actualidad la cifra de cien, orientados a la búsqueda de respuestas a los problemas y necesidades de este sector poblacional, acumulados tanto durante la crisis como en el período anterior a ella.

Lo característico de estos programas es que van encaminados a borrar las desigualdades existentes al interior del grupo infantojuvenil, al abrir nuevas vías de acceso al estudio, en particular a los universitarios, y al trabajo - lo que provoca una fuerte movilidad e integración social - con nuevos enfoques en la recreación y en la educación político-moral de las más nuevas generaciones.

$\mathrm{Al}$ respecto, Fidel ha dicho:

No. Seríamos un ejemplo de vanidad, chovinismo y autosuficiencia si dijéramos que estamos satisfechos. Aun en sociedades como la de $\mathrm{Cu}-$ ba, surgida de una revolución social radical donde el pueblo alcanzó la plena y total igualdad legal y un nivel de educación revolucionaria que echó por tierra gran parte del componente subjetivo de la discriminación, ésta existe todavía de otra forma. La califico como discriminación objetiva, un fenómeno asociado a la pobreza y a un monopolio histórico de los conocimientos.

La Revolución, más allá de los derechos y garantías alcanzados para todos los ciudadanos de cualquier etnia y origen, no ha logrado el mismo éxito en la lucha por erradicar las diferencias en el status social y económico de la población negra del país. Los negros no viven en las mejores casas, se les ve todavía desempeñando trabajos duros y a veces menos remunerados, y son menos los que reciben remesas familiares en moneda exterior que sus compatriotas blancos (Castro, 2006).

En este sentido, de acuerdo con fines específicos, los objetivos generales de la política de juventud que se ejecuta son: rescate de los jóvenes desvinculados del estudio y el trabajo; formación y recalificación laboral y profesional; contribuir a que los niños y jóvenes se apropien de las nuevas tecnologías de la información y la comunicación como parte del proceso de informatización de la sociedad, en marcha; inserción laboral de la juventud; formación de personal especializado; creación de nuevos puestos de trabajo; formación de una cultura general e integral; formación político/ideológica; empleo sano del tiempo 
libre; rescate, renovación y desarrollo de los servicios de educación y salud y rescate y estímulo de la lectura.

La Unión de Jóvenes Comunistas ha sido la principal organización coordinadora y promotora de estos programas, en cuya ejecución y planeamiento participan, además, un conjunto de otros organismos e instituciones estatales, sociales y culturales. En todos los casos de los programas señalados, los recursos proceden del Presupuesto Nacional y de los Fondos de Reserva del Estado. A partir del 2009, los programas han ido pasando a los diferentes organismos centrales del Estado - léase ministerios - cuyos objetivos centrales generales abarquen los de cada programa en particular, sin que éstos pierdan la esencia para la que fueron creados.

Como ejemplo de programas que permiten disminuir las desigualdades sociales - en particular las raciales, asociadas a otras desigualdades como las territoriales y de ocupación del espacio urbano- al interior del sector juvenil y de la población en general, pueden presentarse los siguientes (para una ampliación sobre estos programas, Gómez, 2006).

En el sector educacional: transformaciones en la enseñanza primaria con un maestro por cada 20 alumnos de forma personalizada; transformación de la enseñanza secundaria básica con el maestro general e integral que atiende a un mínimo de estudiantes de forma personalizada; mejoramiento de la alimentación escolar; construcción, ampliación y reparación de escuelas; universalización de la educación superior (extensión de los centros de estudios superiores a todos los municipios del país lo que implica la creación de sedes universitarias municipales); cursos de superación integral para jóvenes desvinculados; curso de superación integral para trabajadores de la industria azucarera; introducción en la educación a todos los niveles y tipos de enseñanza de la computación, la televisión y el video; introducción en la docencia de las teleclases; uso creciente de los medios audiovisuales para fomentar los conocimientos, la cultura y la información, que incluye programas como los siguientes:

Distribución de televisores y videos: creación de dos canales de televisión educativa, así como la apertura de un quinto canal televisivo, por los cuales se transmite una variada e instructiva programación; la transmisión de programas fílmicos, documentales y musicales en los que se resaltan los valores de pueblos del llamado tercer mundo; salas de video club juvenil; salas de televisión en asentamientos rurales.

Programas para promover la consolidación de la cultura artística: formación de instructores de arte; creación de la imprenta Alejo Carpen- 
tier; creación de bibliotecas populares y bibliotecas familiares; programa editorial libertad para proveer a las bibliotecas escolares y públicas de bibliografía de consulta actualizada; desarrollo de las artes plásticas con la creación de 15 escuelas de la especialidad diseminadas por todo el país; creación de la escuela nacional de ballet; creación de un centro productor de dibujos animados; creación de bandas municipales de música.

La informatización de la sociedad comprende la creación de: joven club de computación; palacios de computación; formación de una red entre las universidades del país; creación de la Universidad de las Ciencias Informáticas (UCI).

Relacionados con la atención de la población infanto-juvenil y su desarrollo social: formación de las brigadas universitarias de trabajo social; formación intensiva de trabajadores sociales en cuatro escuelas que preparan jóvenes de todos los territorios del país.

En relación con la salud: formación emergente de enfermería; formación emergente de técnicos de la salud; atención y estudio psicosocial de todos los discapacitados; desarrollo de estudios genéticos de la población con retraso mental; construcción del centro nacional de genética médica; desarrollo de una programa de formación emergente de asesores genéticos; atención a enfermos de fibrosis quísticas.

En relación con la prevención social, se plantea dos objetivos básicos: a) ir al rescate de jóvenes y menores proclives a cometer delitos, b) lograr la transformación de las prisiones en escuelas.

Los propios programas en marcha, en su conjunto y de forma integrada, constituyen una importante obra de prevención social, pues van a la inserción en la sociedad de jóvenes que antes no estudiaban ni trabajaban, asegurándoles superación, formación profesional y trabajo.

En cuanto al segundo objetivo: la extensión del programa audiovisual a los centros penitenciarios; el tratamiento diferenciado al joven recluso; el mejoramiento de las condiciones de vida y trabajo; selección y preparación de los reclusos para cursos de enfermería; desarrollo con la cooperación del Instituto Nacional de Deportes, Educación Física y Recreación (INDER) de cursos de educación física con la población penal; la organización de bibliotecas en las prisiones; ampliación de las posibilidades de estudio hasta el nivel universitario por parte de los reclusos.

Empleo:amparo de los trabajadores disponibles con el 60\% de garantía salarial y el $80 \%$ para los reubicados definitivos; desarrollo de programas de capacitación para recalificar a trabajadores racionalizados; redistribución gradual de la fuerza de trabajo excedente de acuerdo con 
el redimensionamiento y la reestructuración de la economía; aplicación de programas territoriales de empleo integrados al plan de desarrollo económico y social con el propósito de alcanzar las soluciones más convenientes, evitando la concentración de acciones en un mismo lugar y tiempo; elaboración de un diagnóstico de los recursos laborales en cada municipio y provincia y medidas de fomento del empleo; incremento de las posibilidades de empleo, tanto en sector cooperativo, como en el privado; atención especial a los grupos vulnerables, como discapacitados, madres solas, etcétera; incorporación al trabajo de los egresados de las universidades y mantenimiento de la atención a los graduados de técnico medio y protección cuando no fuera posible asegurar su empleo; atención a la creciente incorporación de la mujer al empleo; asegurar el pleno empleo a toda la población, en particular a los jóvenes.

Las principales medidas adoptadas en materia de salud juvenil en el país se encuentran: la aplicación de programas ya tradicionales: atención materno infantil, accidentes para menores de 20 años, prevención y atención a las enfermedades de transmisión sexual, prevención y control del VIH SIDA y atención a los discapacitados; programa nacional de atención estomatológica; programa de atención al adolescente; programa de atención integral a la familia, integrado a la atención primaria de salud; programa de maternidad y paternidad conscientes; prevención y tratamiento de las drogodependencias; proceso de consolidación, reforma y modernización del sistema nacional de salud.

Este conjunto de medidas que conforman los programas, no tienen un carácter estático, sino que constituyen una política dinámica que debe irse adecuando acorde con el desarrollo del país y con los nuevos retos que implican la escalada de crisis - económica, alimentaria, energética y medioambiental - que enfrenta la humanidad y que pone en peligro de extinción a la propia especie humana. En este sentido, toda política social con un alto grado de humanismo que intente una mayor equidad, es un aporte, en el plano interno, a paliar los efectos de esas crisis.

\section{CONCLUSIONeS}

La política general de la Revolución desde el propio triunfo de 1959, ha ido probando la alta efectividad de las políticas universales de equidad para dinamizar procesos acelerados de movilidad social ascendente para los sectores populares y mejorar su acceso a oportunidades de integración social y sus resultados concretos, y con ello, para 
el mejoramiento de los grupos raciales preteridos, mayoritarios, generalmente, dentro de estos sectores.

A pesar de esto existe una permanencia y regeneración intergeneracional de brechas de equidad racializadas, asociadas a condiciones de partida desventajosas que suponen barreras de movilidad social al obstaculizar el aprovechamiento de las oportunidades creadas.

Esto ha implicado la necesidad de combinar instrumentos universales con acciones afirmativas integrales, a las que responden, en gran medida, los programas, que como ejemplo, aquí se han expuestos.

De interés vital resulta el tema racial para un país que construye una perspectiva de equidad enmarcada en una movilidad orientada hacia la integración social e integralidad de la juventud. Las desigualdades raciales persisten en Cuba, y se han hecho más visibles principalmente a partir de la crisis económica de los años 90. Se trata de la consecuencia de la funcionalización de formas de racismo que han permanecido agazapadas en la subjetividad de muchas personas.

Aunque la temática de las relaciones, el prejuicio y la discriminación por color de la piel y sus implicaciones para la sociedad cubana, está siendo investigada por diferentes centros científicos del país, principalmente desde las perspectivas de las ciencias sociales, económicas y pedagógicas, debe continuar profundizándose para poder incidir, con los resultados alcanzados, en su desenmascaramiento y desestructuración.

En general, la temática de las relaciones, el prejuicio y la discriminación por color de la piel, ha ido ocupando espacios públicos, oficiales y alternativos, docentes y barriales, televisivos, científicos y culturales, por lo que se ha ido produciendo un debate público que ha ido resultando en un acorralamiento de este fenómeno tan negativo para el desarrollo de la sociedad cubana.

En este sentido, el país ha acometido una serie de transformaciones que abarca escenarios como el escolar, incluyendo el universitario, el laboral, el de salud, el tecnológico, entre otros, dirigidos a ir eliminando las brechas que fueron quedando durante los primeros treinta años de socialismo en Cuba y que se fueron ampliando y agudizando durante los años 90 . 


\section{REFERENCIAS BIBLIOGRÁFICAS}

AÑÉ, LiA; ÁNGELA FERRIOL y MARIBEL RAMOS (2004): Reforma económica y población en riesgo de Ciudad de la Habana. La Habana: Instituto Nacional de Investigaciones Económicas (inédito).

CASTRO RUZ, Fidel (2006): Cien horas con Fidel: conversaciones con Ignacio Ramonet. La Habana: Oficina de Publicaciones del Consejo de Estado.

Castro Ruz, Fidel (2003): Discurso de Clausura del Congreso de Pedagogía. La Habana: Oficina de Publicaciones del Consejo de Estado.

Colectivo De Autores (2009): Equidad y movilidad social en Cuba. Impactos del reajuste estructural. La Habana: Centro de Investigaciones Psicológicas y Sociológicas (inédito).

(2003): Relaciones raciales en Ciudad de La Habana, Santa Clara y Santiago de Cuba. La Habana: Centro de Antropología (inédito).

CuBA. Biblioteca Nacional «José Martí» (1986): Bibliografía de temas afrocubanos. La Habana: Biblioteca Nacional José Martí.

DOMínGUEZ, M. I. y M. R. DíAz (1997): Reproducción social y acceso a la educación en Cuba. La Habana: Centro de Investigaciones Psicológicas y Sociológicas (inédito).

ESPINA, R. y P. RoDRÍGUEZ (2006): «Raza y desigualdad en la Cuba actual». Temas $\mathrm{N}^{\circ} 45$. La Habana.

GÓMEZ SUÁREZ, LUIS (2006): «La política cubana de juventud y los programas de la Revolución». La Habana: CESJ (inédito).

IZQUIERDO, P. y P. RODRÍGUEZ (2006): «Un acercamiento al estudio de las representaciones raciales entre los estudiante de la Universidad de La Habana». Ponencia presentada en Antropología 06 (inédito).

Partido Comunista de CuBA (1985): Informe central al Congreso. La Habana: Oficina de Publicaciones del Consejo de Estado.

Pérez Álvarez, MARÍA MAgdalenA (1996): «Los prejuicios raciales: sus mecanismos de reproducción». Temas $\mathrm{N}^{\circ} 7$. La Habana.

RodríGueZ, PABlo; ANA GARCÍA y LÁZARA CARRAZANA (2003): Relaciones raciales en la esfera laboral. La Habana: Centro de Antropología (inédito). 